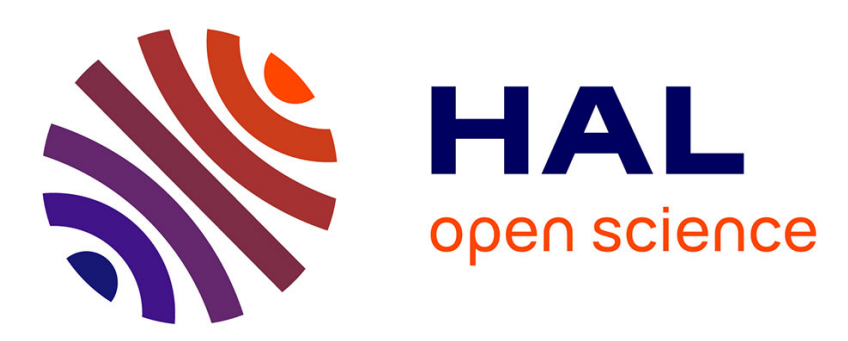

\title{
Approximations of the measurable quantity in diffuse optical problems: theoretical analysis of model deviations
}

\author{
N. Ducros, Anabela da Silva, J.-M. Dinten, F. Peyrin
}

\section{To cite this version:}

N. Ducros, Anabela da Silva, J.-M. Dinten, F. Peyrin. Approximations of the measurable quantity in diffuse optical problems: theoretical analysis of model deviations. Journal of the Optical Society of America. A Optics, Image Science, and Vision, 2008, 25 (5), pp.1174-1180. 10.1364/JOSAA.25.001174 . hal-00872351

\section{HAL Id: hal-00872351 \\ https://hal.science/hal-00872351}

Submitted on 11 Oct 2013

HAL is a multi-disciplinary open access archive for the deposit and dissemination of scientific research documents, whether they are published or not. The documents may come from teaching and research institutions in France or abroad, or from public or private research centers.
L'archive ouverte pluridisciplinaire HAL, est destinée au dépôt et à la diffusion de documents scientifiques de niveau recherche, publiés ou non, émanant des établissements d'enseignement et de recherche français ou étrangers, des laboratoires publics ou privés. 


\title{
Approximations of the measurable quantity in diffuse optical problems: theoretical analysis of model deviations
}

\author{
Nicolas Ducros, ${ }^{1,2, *}$ Anabela da Silva, ${ }^{1}$ Jean-Marc Dinten, ${ }^{1}$ and Françoise Peyrin ${ }^{2}$ \\ ${ }^{1}$ Micro Technologies for Biology and Healthcare Division, Commissariat à l'Énergie Atomique-Laboratoire \\ d'Électronique de Technologie et d'Instrumentation Micro et Nano Technologies, 17 rue des Martyrs, 38054 Grenoble, \\ France \\ ${ }^{2}$ Centre de Recherche et d'Applications en Traitement de l'Images et du Signal-UMR CNRS 5220-INSERM U630, \\ INSA Lyon, Bât. Blaise Pascal, 69621 Villeurbanne Cedex, France \\ *Corresponding author: nicolas.ducros@cea.fr
}

Received November 20, 2007; revised February 16, 2008; accepted March 24, 2008; posted March 25, 2008 (Doc. ID 89873); published April 28, 2008

\begin{abstract}
Photon density and photon flux are widely used to model the measurable quantity in diffuse optical tomography problems. However, it is not these two quantities that are actually measured, but rather the radiance accepted by the detection system. We provide a theoretical analysis of the model deviations related to the choice of the measurable quantity-either photon density or flux. By using the diffusion approximation to the radiative transfer equation and its solution with extrapolated boundary conditions, an exact analytical expression of the measurable quantity has been obtained. This expression has been employed as a reference to assess model deviation when considering the photon density or the photon flux as the measurable quantity. For the case of semi-infinite geometry and for both continuous wave and time domains, we show that the photon density approximates the measurable quantity better than the photon flux. We also demonstrate that the validity of this approximation strongly depends on the optical parameters. (c) 2008 Optical Society of America

OCIS codes: $170.0170,170.3660,170.6920,170.7050,170.6960$.
\end{abstract}

\section{INTRODUCTION}

The diffusion equation (DE) is known to model to good approximation the photon migration processes in biological tissue. Solutions of the DE have been used extensively for the optical characterization of turbid media, in diffuse optical tomography (DOT) $[1,2]$ and fluorescence diffuse optical tomography (FDOT) $[3,4]$. Although one requires expressing the experimentally measurable quantity (MQ), the solutions of the DE are given in terms of photon densities. A question of interest, already addressed by different authors, is to determine the relation between the photon density and the MQ. The two main approaches are (1) the MQ is proportional to the photon density [1,3] and (2) the $\mathrm{MQ}$ is proportional to the derivative of the photon density normal to the boundary, namely, the photon flux $[5,6]$.

Lui et al. [7] suggested that the two approaches are equivalent since the photon density and flux are proportional. This assertion is valid when regarding solutions of the DE obtained with the partial current boundary condition as shown by Martelli et al. [8]. However, the alternative extrapolated boundary condition (EBC) is often applied, since it leads to the derivation of analytical solutions of the DE [6]. These EBC solutions are widely employed and appreciated because of the short computation time they require. In that case, there is no $a$ priori proportionality between photon density and photon flux, and both terms must be taken into account.
Kienle and Patterson [9] first reported improvement in the determination of optical properties when density and flux terms are both considered. The same hybrid expression has been used in the steady-state bioluminescence problem by Comsa et al. [10]. Xu et al. [11] reported enhanced description of continuous wave (CW) light distribution with a distinct expression involving density and flux. Finally, Laidevant et al. [12] derived a hybrid expression for the time-resolved (TR) light distribution. However, the vast majority of authors consider either only photon density or only photon flux. Indeed, it is also claimed that there is no difference in taking one or the other or both terms. For instance, Haskell et al. [13] reported little difference in the simulated MQ when neglecting the density term.

Since deviations between model and measurements can dramatically affect ill-posed DOT and FDOT problems, we propose an evaluation of model deviation due to the choice of MQ. Our evaluation is carried out by comparing the EBC photon density and the EBC photon flux at a surface to a reference MQ. Tomographic schemes consist of reconstructing quantities (absorption, diffusion, fluorescence yield) from surface excitations and measurements. Whatever the quantity to be reconstructed, that implies in practice the calculation of the $\mathrm{MQ}$ of light for any position of excitation within the medium. Consequently, the evaluation has been done simultaneously on a wide set of source positions within the medium. 
Finally, we restrict the study to a semi-infinite geometry, allowing the simplest description of a bounded medium. However, a more complex geometry such as a slab or a parallelepiped can be seen as the combination of semi-infinite media. Such an expansion can be performed by means of the method of images [14]. Therefore, the deviation trends observed in the semi-infinite geometry could be reasonably extrapolated to those in the slab or parallelepiped geometry.

In Section 2, we present the classical analytical expressions of photon density and photon flux used to model MQ. We also detail our reference MQ based on the diffusion approximation and solution of the DE obtained with EBC. We propose to address both the $\mathrm{CW}$ and the TR case. Since the $\mathrm{CW}$ case can be derived from the TR case by integration over all time, the problem is put into equations in the more general time domain.

In Section 3, we evaluate the deviation due to the modeling of the MQ by photon density and photon flux. We emphasize here the necessity to compare the MQ models for a large variety of source positions. Comparisons based only on the temporal shapes of the MQ underestimate the deviation due to neglecting the influence of varying source-detector separation. The evaluation is done in the $\mathrm{CW}$ domain and in the TR domain. We see that CW deviations differ from TR deviations in some situations. Finally, the evaluation is done for several combinations of optical parameters and for several numerical apertures (NA) of the detection system. We observe a strong dependence on some optical parameters.

\section{THEORY}

In this study, we consider a homogeneous semi-infinite medium of absorption coefficient $\mu_{a}\left(\mathrm{~cm}^{-1}\right)$, diffusion constant $D=1 / 3 \mu_{s}^{\prime}(\mathrm{cm})$ as recommended in [15], and refractive index $n$. As depicted in Fig. 1, this medium is surrounded by a nonabsorbing and nondiffusing medium of refractive index $n_{0}$.

\section{A. Photon Density with Extrapolated Boundary Conditions}

The photon density $\phi$ (photon number $/ \mathrm{cm}^{2} / \mathrm{ns}$ ) in a diffusing medium is defined as the integration of the radiance $L$ (photon number $/ \mathrm{cm}^{2} / \mathrm{ns} / \mathrm{sr}$ ) over all solid angles [16]:

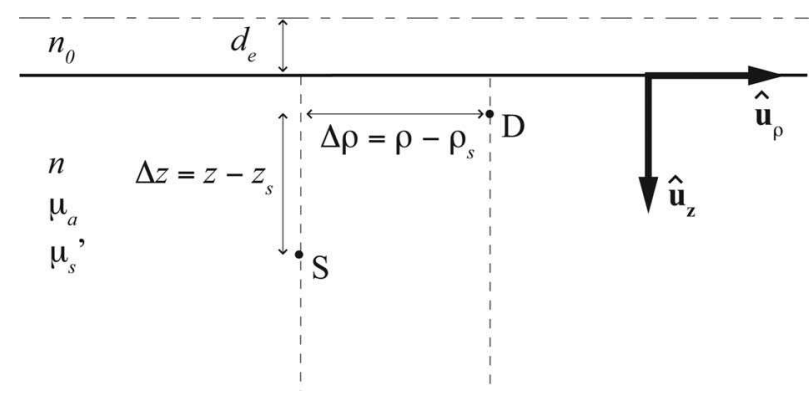

Fig. 1. Position of the problem and notations. The lower semiinfinite medium is absorbing and scattering. The position of the source $\mathrm{S}$ expressed in cylindrical coordinates is $\left(\rho_{s}, z_{s}\right)$, the position of the detector $\mathrm{D}$ is $(\rho, z)$. The relative position between $\mathrm{S}$ and $\mathrm{D}$ is represented by $\Delta z$ and $\Delta \rho$.

$$
\phi(\mathbf{r}, t) \equiv \int_{4 \pi} L(\hat{\mathbf{s}}, \mathbf{r}, t) \mathrm{d} \hat{\mathbf{s}} .
$$

In diffusing media, analytical expressions of EBC photon densities have been well established for simple geometries [6]. In our semi-infinite problem, the photon density $\phi$ at position $\mathbf{r}=\rho \hat{\mathbf{u}}_{\rho}+z \hat{\mathbf{u}}_{\mathrm{z}}$ and time $t(\mathrm{~ns})$ due to the injection of a light pulse at position $\mathbf{r}_{\mathbf{s}}=\rho_{s} \hat{\mathbf{u}}_{\rho}+z_{s} \hat{\mathbf{u}}_{\mathrm{z}}$ and time $t$ $=0$ is given by

$$
\begin{aligned}
\phi\left(\mathbf{r}, \mathbf{r}_{\mathbf{s}}, t\right)= & \frac{\nu}{(4 \pi \nu D t)^{3 / 2}} \exp \left(-\mu_{a} \nu t-\frac{\Delta \rho^{2}}{4 \nu D t}\right) \\
& \times\left[\exp \left(-\frac{\Delta z^{2}}{4 \nu D t}\right)\right. \\
& \left.-\exp \left(-\frac{\left(z+2 d_{e}+z_{s}\right)^{2}}{4 \nu D t}\right)\right]
\end{aligned}
$$

where $\nu(\mathrm{cm} / \mathrm{ns})$ is the speed of light in the medium, $\Delta \rho$ $=\rho-\rho_{s}(\mathrm{~cm})$ and $\Delta z=z-z_{s}(\mathrm{~cm})$ represents the radial and normal source-detector separation. The distance $d_{e}(\mathrm{~cm})$ is the distance of extrapolation away from the physical interface and where the photon density vanishes. The distance of extrapolation is calculated according to [13] and [17], taking into account the index mismatch between the diffusing and the nondiffusing medium (see [18] for further discussion of that distance).

B. Photon Flux with Extrapolated Boundary Conditions The photon flux $F$ (photon number $/ \mathrm{cm}^{3} / \mathrm{ns}$ ) in the direction $\hat{\mathbf{n}}$ is defined from the photon density. Several expressions, differing only in a multiplicative factor, have been used $[6,9]$. Let us keep the simple definition

$$
F(\mathbf{r}, t) \equiv \nabla \phi(\mathbf{r}, t) \cdot \hat{\mathbf{n}} .
$$

The photon flux normal to the boundary is easily given by deriving Eq. (2) along $\hat{\mathbf{u}}_{\mathbf{z}}$. The classical expression is then obtained:

$$
\begin{aligned}
F\left(\mathbf{r}, \mathbf{r}_{\mathbf{s}}, t\right)= & \frac{1}{2 D t(4 \pi \nu D t)^{3 / 2}} \exp \left(-\mu_{a} \nu t-\frac{\Delta \rho^{2}}{4 \nu D t}\right) \\
& \times\left\{\left(z+2 d_{e}+z_{s}\right) \exp \left[-\frac{\left(z+2 d_{e}+z_{s}\right)^{2}}{4 \nu D t}\right]\right. \\
& \left.-\Delta z \exp \left(-\frac{\Delta z^{2}}{4 \nu D t}\right)\right\} .
\end{aligned}
$$

It can be seen from Eq. (2) and Eq. (4), that photon density and photon flux are not proportional. Indeed, the ratio between these two quantities is not a constant and depends notably on the time and on the normal sourcedetector separation.

\section{Measurable Quantity}

The MQ at the surface position $\mathbf{r}=\rho \hat{\mathbf{u}}_{\boldsymbol{\rho}}$ has been rigorously defined by Haskell et al. [13]. It is stated that surface MQ is proportional to the radiance $L$ transmitted from the diffuse medium to the detection system and integrated over the angles accepted by the detection system: 


$$
M Q\left(\mathbf{r}, \mathbf{r}_{\mathbf{s}}, t\right) \propto \int_{\Omega_{d e t}} T_{F}(\hat{\mathbf{s}}) L\left(\mathbf{r}, \mathbf{r}_{\mathbf{s}}, \hat{\mathbf{s}}, t\right) \hat{\mathbf{s}} \cdot \hat{\mathbf{n}}(\mathbf{r}) \mathrm{d} \hat{\mathbf{s}},
$$

where $\Omega_{\text {det }}$ is the solid angle corresponding to the NA of detection, $\hat{\mathbf{s}}$ is a unit vector indicating the direction of radiance, and $\hat{\mathbf{n}}$ is a unit vector indicating the direction of detection. We consider normal detection, leading to $\hat{\mathbf{n}}=$ $-\hat{\mathbf{z}}$. All these parameters are represented in Fig. 2. $T_{F}$ is the Fresnel transmission coefficient for unpolarized light given by

$$
\begin{aligned}
T_{F}(\theta)= & 1-\frac{1}{2}\left(\frac{n \cos \theta^{\prime}-n_{0} \cos \theta}{n \cos \theta^{\prime}+n_{0} \cos \theta}\right)^{2} \\
& -\frac{1}{2}\left(\frac{n \cos \theta-n_{0} \cos \theta^{\prime}}{n \cos \theta+n_{0} \cos \theta^{\prime}}\right)^{2}
\end{aligned}
$$

where $\theta$ is the angle of incidence from within the medium and $\theta^{\prime}$ is the refracted angle outside the medium (see Fig. 2) satisfying the Snell-Descartes law $n_{0} \sin \theta^{\prime}=n \sin \theta$ [13].

To go further, the radiance $L$ can be expanded in terms of $\phi$ according to the diffusion approximation [16]:

$$
L\left(\mathbf{r}, \mathbf{r}_{\mathbf{s}}, \hat{\mathbf{s}}, t\right)=\frac{1}{4 \pi} \phi\left(\mathbf{r}, \mathbf{r}_{\mathbf{s}}, t\right)-\frac{3 D}{4 \pi} \nabla \phi\left(\mathbf{r}, \mathbf{r}_{\mathbf{s}}, t\right) \cdot \hat{\mathbf{s}} .
$$

Substituting Eq. (7) in Eq. (5) and after some algebra (see [16] and [19] for details on the derivation) we derive the general form

$$
M Q\left(\mathbf{r}, \mathbf{r}_{\mathbf{s}}, t\right) \propto \phi\left(\mathbf{r}, \mathbf{r}_{\mathbf{s}}, t\right)+3 D C F\left(\mathbf{r}, \mathbf{r}_{\mathbf{s}}, t\right) .
$$

The general Eq. (8) describes the MQ as the weighted sum of a density term and a flux term. The weight coefficients $C$ give the relative amount of photon density and flux that have to be considered. This coefficient depends only on refractive indices $n$ and $n_{0}$ and on the NA of the detection system, since they are analytically given by

$$
C=\frac{\int_{0}^{\theta_{m}} T_{F}\left(\theta^{\prime}\right) \sin \theta^{\prime}\left(1-n_{0}^{2} / n^{2} \sin ^{2} \theta^{\prime}\right) \mathrm{d} \theta^{\prime}}{\int_{0}^{\theta_{m}} T_{F}\left(\theta^{\prime}\right) \sin \theta^{\prime}\left(1-n_{0}^{2} / n^{2} \sin ^{2} \theta^{\prime}\right)^{1 / 2} \mathrm{~d} \theta^{\prime}},
$$

where $\theta_{m}=\arcsin (\mathrm{NA})$ represents the angle of maximal acceptance of the detection system. Note that the integra-

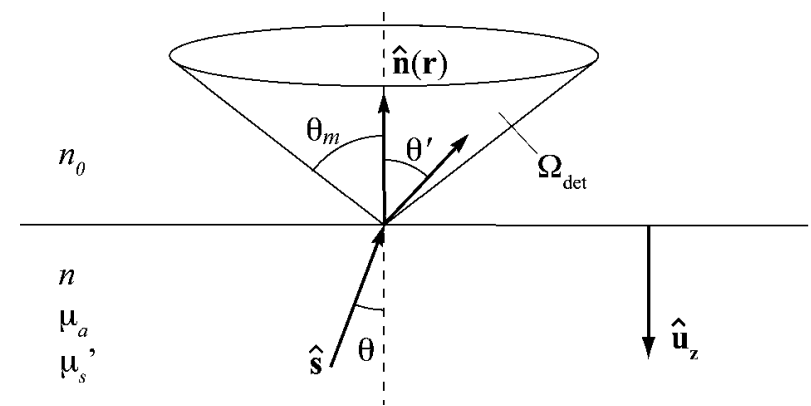

Fig. 2. Determination of the MQ. $\hat{\mathbf{s}} \cdot \hat{\mathbf{n}}=\cos \theta . \theta$ and $\theta^{\prime}$ are related through the Snell-Descartes law. The radiance is integrated over all refracted angles within the acceptance of the detection system. All $\theta^{\prime}$ smaller than $\theta_{m}$ must be considered.
Table 1. Indicating Values of Coupling Coefficients for Different NA and Refractive Indices $n^{a}$

\begin{tabular}{lllll}
\hline & \multicolumn{5}{c}{$n$} \\
\cline { 2 - 5 } NA & 1.33 & 1.4 & 1.5 & 1.54 \\
\hline 0.2 & 0.994 & 0.995 & 0.996 & 0.996 \\
0.5 & 0.963 & 0.967 & 0.971 & 0.973 \\
0.7 & 0.925 & 0.933 & 0.942 & 0.945 \\
0.9 & 0.865 & 0.879 & 0.895 & 0.901 \\
\hline
\end{tabular}

${ }^{a}$ Here $n_{0}$ is set to 1 .

tion is performed on refracted angles corresponding to photons leaving the medium.

Values of the weight coefficient $C$ for different NAs and refractive indices are gathered in Table 1. Equation (8) generalizes for any numerical aperture and refractive index the hybrid expressions proposed by different authors. The expression of Kienle and Patterson [9] and similar expressions are identical to Eq. (8) under conditions of large NA. The expression proposed by Laidevant et al. [12] and similar expressions can be retrieved considering a low NA, for which $C$ tends to 1 .

\section{MODEL DEVIATIONS}

\section{A. Continuous Wave Domain}

In the CW domain, we propose to evaluate the following deviations:

$$
\epsilon_{d, C W}\left(\mathbf{r}, \mathbf{r}_{\mathbf{s}}\right)=\frac{\int \alpha \phi\left(\mathbf{r}, \mathbf{r}_{\mathbf{s}}, t\right) \mathrm{d} t-\int M Q\left(\mathbf{r}, \mathbf{r}_{\mathbf{s}}, t\right) \mathrm{d} t}{\int M Q\left(\mathbf{r}, \mathbf{r}_{\mathbf{s}}, t\right) \mathrm{d} t}
$$

$$
\epsilon_{f, C W}\left(\mathbf{r}, \mathbf{r}_{\mathbf{s}}\right)=\frac{\int \beta F\left(\mathbf{r}, \mathbf{r}_{\mathbf{s}}, t\right) \mathrm{d} t-\int M Q\left(\mathbf{r}, \mathbf{r}_{\mathbf{s}}, t\right) \mathrm{d} t}{\int M Q\left(\mathbf{r}, \mathbf{r}_{\mathbf{s}}, t\right) \mathrm{d} t} .
$$

The model deviation due to the use of the photon density as the MQ is given by Eq. (10a). The model deviation due to the use of photon flux as the MQ is given by Eq. (10b). The two quantities $\epsilon_{d, C W}$ and $\epsilon_{f, C W}$ can be analytically determined by integration of Eq. (2) and Eq. (4) and use of Eq. (8). Analytical expression of the integrals of Eq. (2) and Eq. (4) can be found in [6]. CW deviations depend on the depth of the source $\Delta z=-z_{s}$ and on the radial sourcedetector separation $\Delta \rho$. The coefficients of proportionality $\alpha$ and $\beta$ are calibrated such that $\mathrm{CW}$ deviations are null for large source-detector separations. For such long distances of propagation, the photon density and the photon flux are proportional. This assertion is proved in Appen$\operatorname{dix}$ A.

An illustration of $\mathrm{CW}$ deviations is given on Fig. 3. To investigate tomographic conditions, we represent deviations for 143 different source-detector relative positions. 

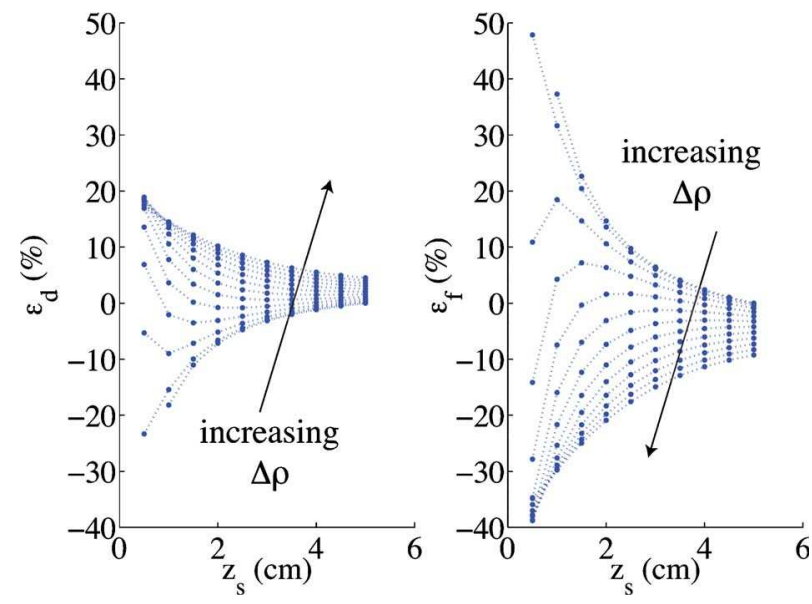

Fig. 3. (Color online) CW deviations in tomographic conditions. MQ is approximated by the photon density (left) and by the photon flux (right). $z_{s}$ varies from $1 / \mu_{s}^{\prime}$ to $5 \mathrm{~cm}$ and $\Delta \rho$ from 0 to $4 \mathrm{~cm}$. Here $\mu_{a}=0.05 \mathrm{~cm}^{-1}, \mu_{s}^{\prime}=2 \mathrm{~cm}^{-1}, n=1.54$, and NA=0.7. The closer to the surface the source is, the larger the deviations are.

Table 2. CW Deviations at $z_{s}=1 / \mu_{s}^{\prime} \mathrm{cm}$ for $\mu_{a}=0.05 \mathrm{~cm}^{-1}$ and $\mathrm{NA}=0.7$

\begin{tabular}{ccrrrr}
\hline & & \multicolumn{2}{c}{$\epsilon_{d, C W}(\%)$} & \multicolumn{2}{c}{$\epsilon_{f, C W}(\%)$} \\
\cline { 3 - 6 }$\mu_{s}^{\prime}(\mathrm{cm})^{-1}$ & $n$ & \multicolumn{1}{c}{ Min } & Max & \multicolumn{1}{c}{ Min } & Max \\
\hline 2 & 1.33 & -18.9 & 14.1 & -21.9 & 29.4 \\
5 & 1.33 & 1.2 & 9.0 & -17.0 & -2.0 \\
20 & 1.33 & 0.5 & 5.5 & -9.5 & -0.8 \\
2 & 1.54 & -23.3 & 18.9 & -38.8 & 47.9 \\
5 & 1.54 & -2.4 & 12.0 & -29.7 & 5.9 \\
20 & 1.54 & 2.2 & 7.5 & -20.3 & -0.8 \\
\hline
\end{tabular}

The smallest $z_{s}$ investigated was chosen equal to $1 / \mu_{s}^{\prime}$. This choice is based on external laser beam excitations, as this type of excitation is commonly modeled by a source of light positioned at depth $1 / \mu_{s}^{\prime}$ below the surface [5]. We investigate $z_{s}$ up to $5 \mathrm{~cm}$ and $\Delta \rho$ up to $4 \mathrm{~cm}$. These dimensions are large enough for DOT and FDOT problems.

It can be seen that for both photon density and flux, the shallower the source is, the larger the deviations are. In particular, the photon density and the photon flux due to a surface excitation differ significantly from the MQ. These deviations will be of particular consequence with DOT and FDOT problems for which the quantity to be reconstructed (absorption, diffusion, or fluorescence yield) is close to the surface. Interestingly, it can be noted that choosing photon density as the MQ leads to lower deviations than choosing photon flux. We calculated the same deviation pictures as in Fig. 3 for several $\mu_{a}$ $\in[0.02,0.2] \mathrm{cm}^{-1}$, for several $\mu_{s}^{\prime} \in[2,20] \mathrm{cm}^{-1}$, for several $n \in[1.33,1.54]$ (from water to epoxy resin), and for several NA $\in[0.2,0.9]$. These sets of parameters cover most of the situations encountered in DOT and FDOT problems.

The same trends in deviations with increased or reduced amplitudes were observed for all these configurations. Some interesting results for $z_{s}=1 / \mu_{s}^{\prime} \mathrm{cm}$ are reported in Table 2. We observed that the deviations were mainly amplified by the parameter $\mu_{s}^{\prime}$. A low-diffusing medium leads to large deviations. Deviations are accentu- ated by high values of $n$, especially when the photon flux is assumed to describe the MQ. Finally, deviations are affected to a lower extent by the parameters $\mu_{a}$ and NA.

\section{B. Time Domain}

In the time domain, we introduce the following deviations:

$$
\begin{aligned}
& \epsilon_{d, T R}\left(\mathbf{r}, \mathbf{r}_{\mathbf{s}}, t\right)=\frac{\alpha \phi\left(\mathbf{r}, \mathbf{r}_{\mathbf{s}}, t\right)-M Q\left(\mathbf{r}, \mathbf{r}_{\mathbf{s}}, t\right)}{M Q\left(\mathbf{r}, \mathbf{r}_{\mathbf{s}}, t\right)} \\
& \epsilon_{f, T R}\left(\mathbf{r}, \mathbf{r}_{\mathbf{s}}, t\right)=\frac{\beta F\left(\mathbf{r}, \mathbf{r}_{\mathbf{s}}, t\right)-M Q\left(\mathbf{r}, \mathbf{r}_{\mathbf{s}}, t\right)}{M Q\left(\mathbf{r}, \mathbf{r}_{\mathbf{s}}, t\right)}
\end{aligned}
$$

In the same way, the model deviations due to the use of photon density as a model of the MQ is given by Eq. (11a). The model deviation due to the use of flux as a model of the MQ is given by Eq. (11b). The two quantities $\epsilon_{d, T R}$ and $\epsilon_{f, T R}$ can be easily derived using Eq. (2), Eq. (4), and Eq. (8). TR deviations depend also on the depth of the source $\Delta z=-z_{s}$ and on the time $t$ but, contrary to the CW case, not on the radial source-detector separation $\Delta \rho$.

An illustration of TR deviations is given in Fig. 4. We chose the same relative source-detector positions as in the CW domain (see subsection 3.A). The coefficients of proportionality $\alpha$ and $\beta$ calibrated for the CW study were also used in the time domain. For both photon density

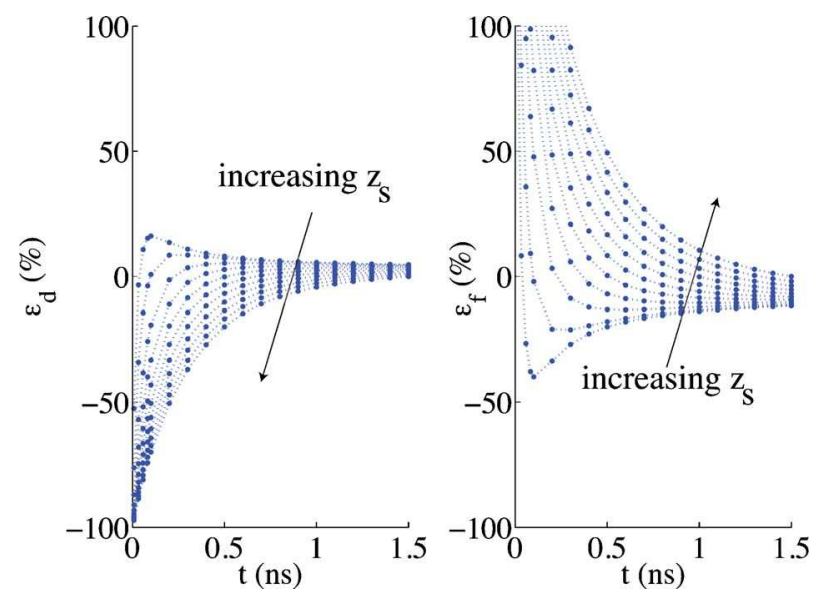

Fig. 4. (Color online) TR deviations in tomographic conditions. MQ is approximated by the photon density (left) and by the photon flux (right). $z_{s}$ varies from $1 / \mu_{s}^{\prime}$, to $5 \mathrm{~cm}$. TR deviations do not depend on $\Delta \rho$. Here $\mu_{a}=0.05 \mathrm{~cm}^{-1}, \mu_{s}^{\prime}=5 \mathrm{~cm}^{-1}, n=1.54$, and NA $=0.7$. The earlier the time, the larger the deviations.

Table 3. TR Deviations at $\mu_{s}^{\prime}=5 \mathrm{~cm}^{-1}, \mu_{a}=0.05 \mathrm{~cm}^{-1}$, and $\mathrm{NA}=0.7$

\begin{tabular}{clrrrrr}
\hline & & \multicolumn{2}{c}{$\epsilon_{d, T R}(\%)$} & & \multicolumn{2}{c}{$\epsilon_{f, T R}(\%)$} \\
\cline { 3 - 4 } \cline { 6 - 7 }$t(\mathrm{~ns})$ & $n$ & Min & Max & & Min & Max \\
\hline 0.1 & 1.33 & -70.0 & 11.9 & & -20.4 & 105.6 \\
0.1 & 1.4 & -64.9 & 14.0 & & -27.6 & 127.5 \\
0.1 & 1.54 & -61.5 & 16.1 & & -40.0 & 173.1 \\
1.0 & 1.33 & -1.2 & 2.9 & & -5.0 & 2.0 \\
1.0 & 1.4 & -1.9 & 3.7 & & -7.3 & 3.8 \\
1.0 & 1.54 & -4.2 & 5.5 & & -13.9 & 10.5 \\
\hline
\end{tabular}


and flux, the smaller $t$ is, the larger the deviations. At early time, deviations are particularly high, especially for deep sources and when the MQ is modeled by the photon flux. Here again, it can be seen that the use of photon density rather than photon flux results in smaller deviations. Analogously to the CW case, deviations were evaluated for several $\mu_{a} \in[0.02,0.2] \mathrm{cm}^{-1}$, several $\mu_{s}^{\prime} \in[2,20] \mathrm{cm}^{-1}$, several $n \in[1.33,1.54]$, and several $\mathrm{NA} \in[0.2,0.9]$. As for the $\mathrm{CW}$ domain, the trends of deviations remain the same for all configurations but exhibit increased or reduced amplitudes. In the TR case the main amplifying parameter is found to be $n$. The larger $n$ is, the larger the deviations are whether photon density if or photon flux is considered. Examples of deviations for different $n$ are shown in Table 3. Secondarily, low $\mu_{s}^{\prime}$ leads to larger deviations. The parameters $\mu_{a}$ and NA have limited impact.

\section{DISCUSSION AND CONCLUSION}

In this study, we analyzed the impact of the approximation of the measurable quantity. The main contribution of this work was to show that EBC photon density better describes the measurable quantity than EBC photon flux in DOT and FDOT according to the diffusion approximation. We saw that model deviations due to the approximation of the MQ can be reduced by a factor of 2 when photon density is preferred to photon flux. That conclusion was found to be true for $\mathrm{CW}$ and TR problems for a wide range of optical properties. In addition, it, was proved that the degree of accuracy of approximating the MQ to the photon density strongly depends on the values of the optical parameters.

More precisely, we found for $\mu_{s}^{\prime}=5 \mathrm{~cm}^{-1}$ that the CW photon density differs by less than $15 \%$ from the MQ whatever source position, absorption, refractive index, or numerical aperture is considered. We identified that $\mathrm{CW}$ deviations are larger when the source is close to the surface and also larger if the medium is less diffusing. When the propagation distances are short and when the diffusion processes are limited, the directive (flux) component of the MQ differs significantly from the isotopic (density) component.

In the time domain, we found at $t=1 \mathrm{~ns}$ that the TR photon density deviation from the $\mathrm{MQ}$ is less than $15 \%$ whatever source depth, absorption, diffusion, refractive index or NA is considered. We observed that the largest TR deviations occur at early time and are negative for the photon density (underestimation of the $\mathrm{MQ}$ ) and positive for the photon flux (overestimation of the MQ). We investigated how large these deviations are by deriving the limit of $\epsilon_{d, T R}$ and $\epsilon_{f, T R}$ when $t$ tends to zero (see Appendix $B$ for details). It was shown that using the photon density results in a $100 \%$ underestimation of the $\mathrm{MQ}$ at early time. It was also shown that using photon flux results in an overestimation of the MQ at early time. The amplitude of this overestimation depends on the optical properties of the medium and the NA of the detection system according to formula Eq. (B6). For all the situations investigated in the present paper, the amplitude of the early time photon flux overestimation is always larger than the amplitude of the early time photon density underestimation.
In the time domain, we also established that the stronger $n$ is, the larger the deviations are. This strong index dependency, enhanced at early time, was not observed in the CW domain. The index mismatch has high implications in terms of extrapolation distance. Although the implications in terms of extrapolation distance are the same in the CW domain, the dependency is limited in that domain. The determining aspect of the index dependency may be explained in terms of speed of light in the medium. Indeed, the speed of light affects only the time domain since it cancels out in the $\mathrm{CW}$ domain where phenomena are integrated over time.

In the situations investigated, the NA of the detection system is not a crucial parameter. However, we would like to point out that the influence of this parameter grows sensitive when a low-diffusing medium is considered, as already noted by Martelli et al. [8] with partial current boundary conditions.

In this paper we identified and quantified model deviations due to the modeling of the MQ. Our analysis is based entirely on the diffusion approximation. It may be interesting to note that the situations for which the use of photon density to model the $\mathrm{MQ}$ becomes questionable are situations for which the diffusion approximation starts to be invalid. Indeed, the diffusion approximation breaks down when low-diffusing media, near-to-surface source positions, and early time are examined. It can be postulated that the diffusion approximation can hold in a wider range of validity when the exact $\mathrm{MQ}$ expression given in Eq. (5) is used. Further studies with experimental measurements could enlarge the discussion and provide an answer to this question.

\section{APPENDIX A: LIMIT OF THE RATIO BETWEEN PHOTON DENSITY AND PHOTON FLUX FOR DEEP SOURCE OF LIGHT IN THE CONTINUOUS WAVE DOMAIN}

To evaluate

$$
L_{\infty} \equiv \lim _{z_{s \rightarrow+\infty}} \frac{\int \phi\left(\mathbf{r}, \mathbf{r}_{\mathbf{s}}, t\right) \mathrm{d} t}{\int F\left(\mathbf{r}, \mathbf{r}_{\mathbf{s}}, t\right) \mathrm{d} t},
$$

we make use of analytical expressions of $\int \phi\left(\mathbf{r}, \mathbf{r}_{\mathbf{s}}, t\right) \mathrm{d} t$ and $\int F\left(\mathbf{r}, \mathbf{r}_{\mathbf{s}}, t\right) \mathrm{d} t$ given by Eqs. (12) and (15) of [20]. With our notations, the two equations are rewritten

$$
\begin{aligned}
\int \phi\left(\mathbf{r}, \mathbf{r}_{\mathbf{s}}, t\right) \mathrm{d} t & =\frac{1}{4 \pi D}\left[\frac{\exp \left(-k r_{1}\right)}{r_{1}}-\frac{\exp \left(-k_{2}\right)}{r_{2}}\right], \\
\int \phi\left(\mathbf{r}, \mathbf{r}_{\mathbf{s}}, t\right) \mathrm{d} t & =\frac{1}{4 \pi D}\left[\frac{\left(z_{s}+2 d_{e}\right)\left(k r_{2}+1\right)}{r_{2}^{3}} \exp \left(-k r_{2}\right)\right. \\
& \left.+\frac{z_{s}\left(k r_{1}+1\right)}{r_{1}^{3}} \exp \left(-k r_{1}\right)\right],
\end{aligned}
$$

where $r_{1}^{2}=\Delta \rho^{2}+z_{s}^{2}, r_{2}^{2}=\Delta \rho^{2}+\left(2 d_{e}+z_{s}\right)^{2}$, and $k=\left(\mu_{a} / D\right)^{1 / 2}$. With no loss of generality when $z_{s}$ tends to infinity, $\Delta \rho$ is 
set to zero in Eqs. (A2a) and (A2b). Then the two quantities are factored into the form

$$
\begin{aligned}
& \int \phi\left(\mathbf{r}, \mathbf{r}_{\mathbf{s}}, t\right) \mathrm{d} t=\frac{1}{4 \pi D} \frac{\exp \left(-k z_{s}\right)}{z_{s}}\left[1-\exp \left(-2 d_{e} k\right)\right] A\left(\mathbf{r}, \mathbf{r}_{\mathbf{s}}\right), \\
& \int F\left(\mathbf{r}, \mathbf{r}_{\mathbf{s}}, t\right) \mathrm{d} t=\frac{k}{4 \pi D} \frac{\exp \left(-k z_{s}\right)}{z_{s}}\left[1+\exp \left(-2 d_{e} k\right)\right] B\left(\mathbf{r}, \mathbf{r}_{\mathbf{s}}\right),
\end{aligned}
$$

where the terms $A$ and $B$ both tend to one when $z_{s}$ tends to infinity. Taking the ratio between $\int \phi\left(\mathbf{r}, \mathbf{r}_{\mathbf{s}}, t\right) \mathrm{d} t$ and $\int F\left(\mathbf{r}, \mathbf{r}_{\mathbf{s}}, t\right) \mathrm{d} t$ in the form of Eqs. (A3a) and (A3b) leads to the derivation of the finite limit as

$$
L_{\infty}=\frac{1-\exp \left(-2 d_{e} k\right)}{1+\exp \left(-2 d_{e} k\right)} \frac{1}{k} .
$$

$L_{\infty}$ has the dimensions of length. The strict positivity of $d_{e}$ and $k$ guarantee that $L_{\infty}$ is also positive.

\section{APPENDIX B: BOUNDS OF DEVIATIONS IN THE TIME DOMAIN}

In order to evaluate how large the deviations are at early time, we are interested in deriving the following quantities:

$$
\begin{aligned}
& \lim _{t \rightarrow 0^{+}} \epsilon_{d, T R}\left(\mathbf{r}, \mathbf{r}_{\mathbf{s}}, t\right)=\lim _{t \rightarrow 0^{+}} \alpha \frac{\phi\left(\mathbf{r}, \mathbf{r}_{\mathbf{s}}, t\right)}{M Q\left(\mathbf{r}, \mathbf{r}_{\mathbf{s}}, t\right)}-1, \\
& \lim _{t \rightarrow 0^{+}} \epsilon_{f, T R}\left(\mathbf{r}, \mathbf{r}_{\mathbf{s}}, t\right)=\lim _{t \rightarrow 0^{+}} \beta \frac{F\left(\mathbf{r}, \mathbf{r}_{\mathbf{s}}, t\right)}{M Q\left(\mathbf{r}, \mathbf{r}_{\mathbf{s}}, t\right)}-1 .
\end{aligned}
$$

To derive the two limits in Eq. (B1a) and Eq. (B1b), we rewrite Eqs. (2), (4), and (8) in the form

$$
\begin{gathered}
\phi\left(\mathbf{r}, \mathbf{r}_{\mathbf{s}}, t\right)=\frac{\nu}{(4 \pi \nu D t)^{3 / 2}} \exp \left(-\frac{\Delta \rho+z_{s}^{2}}{4 \nu D t}\right) I\left(\mathbf{r}, \mathbf{r}_{\mathbf{s}}, t\right) \\
F\left(\mathbf{r}, \mathbf{r}_{\mathbf{s}}, t\right)=\frac{z_{s} / 2 D}{(4 \pi \nu D t)^{3 / 2}} \frac{1}{t} \exp \left(-\frac{\Delta \rho+z_{s}^{2}}{4 \nu D t}\right) J\left(\mathbf{r}, \mathbf{r}_{\mathbf{s}}, t\right) \\
M Q\left(\mathbf{r}, \mathbf{r}_{\mathbf{s}}, t\right)=\frac{3 C z_{s} / 2}{(4 \pi \nu D t)^{3 / 2}} \frac{1}{t} \exp \left(-\frac{\Delta \rho+z_{s}^{2}}{4 \nu D t}\right) K\left(\mathbf{r}, \mathbf{r}_{\mathbf{s}}, t\right)
\end{gathered}
$$

where the three terms $I, J$, and $K$ tend to one when $t$ tends to $0^{+}$. Upon substitution of Eq. (B2a) and Eq. (B2c) into Eq. (B1a) and substitution of Eq. (B2b) and Eq. (B2c) into Eq. (B1b), we obtain the following limits:

$$
\lim _{t \rightarrow 0^{+}} \epsilon_{d, T R}\left(\mathbf{r}, \mathbf{r}_{\mathbf{s}}, t\right)=-1,
$$

$$
\lim _{t \rightarrow 0^{+}} \epsilon_{f, T R}\left(\mathbf{r}, \mathbf{r}_{\mathbf{s}}, t\right)=\frac{\beta}{3 C D}-1 .
$$

Using the fact that $\beta$ is calibrated in the $\mathrm{CW}$ domain such that $\epsilon_{d, C W}\left(\mathbf{r}, \mathbf{r}_{\mathbf{s}}\right)$ tends to zero when $z_{s}$ tends to infinity, we can write

$$
\lim _{z_{s} \rightarrow \infty} \beta \frac{\int F\left(\mathbf{r}, \mathbf{r}_{\mathbf{s}}, t\right) \mathrm{d} t}{\int M Q\left(\mathbf{r}, \mathbf{r}_{\mathbf{s}}, t\right) \mathrm{d} t}-1=0 .
$$

With expansion of $M Q$ in terms of $\phi$ and $F$ and use of the definition of $L_{\infty}$ given by Eq. (A1), we derive the simple expression

$$
\beta=L_{\infty}+3 C D
$$

After substitution of Eq. (B5) into Eq. (B3b), we finally obtain

$$
\lim _{t \rightarrow 0^{+}} \epsilon_{f, T R}\left(\mathbf{r}, \mathbf{r}_{\mathbf{s}}, t\right)=\frac{L_{\infty}}{3 C D}>0 .
$$

\section{ACKNOWLEDGMENTS}

This work has been supported by the Région Rhône-Alpes in the context of project 13M "Multiscale medical imaging and modeling: from the small animal to the human being" of cluster ISLE.

\section{REFERENCES}

1. D. A. Boas, M. A. O'Leary, B. Chance, and A. G. Yodh, "Scattering of diffuse photon density waves by spherical inhomogeneities within turbid media: analytic solution and applications," Proc. Natl. Acad. Sci. U.S.A. 91, 4887-4891 (1994).

2. S. R. Arridge, "Optical tomography in medical imaging," Inverse Probl. 15, R41-R93 (1999).

3. X. D. Li, M. A. O'Leary, D. A. Boas, and B. Chance, "Fluorescent diffuse photon density waves in homogeneous and heterogeneous turbid media: analytic solutions and applications," Appl. Opt. 35, 3746-3758 (1996).

4. E. M. Sevick-Muraca, E. Kuwana, A. Godavarty, J. P. Houston, A. B. Thompson, and R. Roy, "Near infrared fluorescence imaging and spectroscopy in random media and tissues," in Biomedical Photonics Handbook, J. VoDinh, ed. (CRC Press, 2003), Chap. 33.

5. M. S. Patterson, B. Chance, and B. C. Wilson, "Time resolved reflectance and transmittance for the noninvasive measurement of tissue optical-properties," Appl. Opt. 28, 2331-2336 (1989).

6. S. R. Arridge, M. Cope, and D. T. Delpy, "The theoretical basis for the determination of optical pathlengths in tissue-temporal and frequency-analysis," Phys. Med. Biol. 37, 1531-1560 (1992)

7. F. Liu, K. M. Yoo, and R. R. Alfano, "Should the photon flux or the photon density be used to describe the temporal profiles of scattered ultrashort laser pulses in random media?" Opt. Lett. 18, 432-434 (1993).

8. F. Martelli, A. Sassaroli, G. Zaccanti, and Y. Yamada, "Properties of the light emerging from a diffusive medium: angular dependence and flux at the external boundary," Phys. Med. Biol. 44, 1257-1275 (1999). 
9. A. Kienle and M. S. Patterson, "Improved solutions of the steady-state and the time-resolved diffusion equations for reflectance from a semi-infinite turbid medium," J. Opt. Soc. Am. A 14, 246-254 (1997).

10. D. C. Comsa, T. J. Farrell, and M. S. Patterson, "Quantification of bioluminescence images of point source objects using diffusion theory models," Phys. Med. Biol. 51, 3733-3746 (2006).

11. T. Xu, C. P. Zhang, G. Y. Chen, J. G. Tian, G. Y. Zhang, and C. M. Zhao, "Theoretical and experimental study of the intensity distribution in biological tissues," Chin. Phys. 14, 1813-1820 (2005).

12. A. Laidevant, A. da Silva, M. Berger, and J. M. Dinten, "Effects of the surface boundary on the determination of the optical properties of a turbid medium with timeresolved reflectance," Appl. Opt. 45, 4756-4764 (2006).

13. R. C. Haskell, L. O. Svaasand, T.-T. Tsay, T.-C. Feng, M. S. McAdams, and B. J. Tromberg, "Boundary conditions for the diffusion equation in radiative transfer," J. Opt. Soc. Am. A 11, 2727-2741 (1994).
14. A. Kienle, "Light diffusion through a turbid parallelepiped,” J. Opt. Soc. Am. A 22, 1883-1888 (2005).

15. R. Pierrat, L. J. Greffet, and R. Carminati, "Photon diffusion coefficient in scattering and absorbing media," J. Opt. Soc. Am. A 23, 1106-1110 (2006)

16. A. Ishimaru, Wave Propagation and Scattering in Random Media (Academic, 1978).

17. J. D. Moulton, "Diffusion modelling of picosecond pulse propagation in turbid media," Master's thesis (McMaster University, Hamilton, Ontario, 1990).

18. R. Aronson, "Boundary conditions for diffusion of light." J. Opt. Soc. Am. A 12, 2532-2539 (1995).

19. J. Ripoll, M. Nieto-Vesperinas, S. R. Arridge, and H. Dehghani, "Boundary conditions for light propagation in diffusive media with nonscattering regions," J. Opt. Soc. Am. A 17, 1671-1681 (2000)

20. T. Farrell, M. Patterson, and B. Wilson, "A diffusion theory model of spatially resolved, steady-state diffuse reflectance for the noninvasive determination of tissue optical properties in vivo," Med. Phys. 19, 879-888 (1992). 